\title{
Quantum Energy Teleportation in Spin Chain Systems
}

\author{
Masahiro Hotta \\ Department of Physics, Faculty of Science, Tohoku University, \\ Sendai, 980-8578, Japan \\ hotta@tuhep.phys.tohoku.ac.jp
}

\begin{abstract}
We propose a protocol of quantum energy teleportation that transports energy in spin chains to distant sites by only local operations and classical communication. The protocol uses ground-state entanglement and localized negative-energy excitation, and the energy is teleported without breaking any physical laws including causality and local energy conservation.
\end{abstract}

Keywords: entanglement, quantum teleportation, spin chain, quantum information theory, condensed matter physics 


\section{Introduction}

Quantum entanglement opens the door to several interesting quantum phenomena, including quantum teleportation (QT) [1], by which an unknown quantum state can be teleported to a distant place by local operations and classical communication (LOCC). However, QT cannot transport the excitation energy of the transported states. For example, let us imagine that Alice sends to Bob a spin-up state of a spin in an external uniform magnetic field parallel to the $\mathrm{z}$ axis. The Hamiltonian is given by

$$
H_{s}=g \sigma_{z}
$$

with a positive constant $g$. The ground state of the spin is the spin-down state with energy $-g$ and the energy of the excited spin-up state is $+g$. A contracted state at Alice or Bob of a Bell pair shared by Alice and Bob for QT is the maximal entropy state, which has zero energy on average. Therefore, both Alice and Bob must first supply energy $+g$ to their spins on average to create the Bell pair from two spins in the ground state by a global operation. Bob has to supply an additional $+g$ energy to his spin to receive the spin-up state from Alice's site by QT. Hence, the total energy input at Bob's site is given by $+2 g$, which should be locally prepared by Bob.

Does the above-described protocol of QT imply that energy cannot be transported by LOCC? Amazingly, the answer is "no". Quantum mechanics allows energy transport by LOCC. In this paper, we propose a protocol of quantum energy teleportation (QET) in spin chains which transports energy only by LOCC, using local excitations with negative energy and groundstate entanglement, thereby respecting fundamental physical laws including causality and local energy conservation.

Spin chains are composed of many spins arrayed in one dimension. Shortrange interactions exist between the spins, and the Hamiltonian is given by a sum of these local interaction terms. Due to the interactions, complicated entanglement among the spins can occur even in the ground state. Spin chains have recently attracted much attention in the context of quantum information theory because they can be applied to the short transmission of quantum states [2]. Spin-chain entanglement is also helpful for investigating the complicated physical properties of the ground state of spin chains [3]. 
Negative localized energy plays an essential role in the protocol. In this paper, we define the zero values of energy density by the expectation values of the ground state. We then consider why there are negative-energy-density regions in the spin chains. In quantum physics, there remain local quantum fluctuations even in the ground states of spin chains. By linearly superposing the eigenstates of the total Hamiltonian, we can suppress the quantum fluctuations more strongly in a local region via quantum interference, as compared with the ground state. Taking a definition such that the energy density in the ground state is zero, the energy density becomes negative in a region where the quantum fluctuation is more greatly suppressed. We might be concerned that there are states with energy values lower than the ground state. However, it should be stressed that, even though some regions have negative energy density, the total energy of the system is always nonnegative. Hence, there exist no states with energy lower than the ground states. Negative energy effects in relativistic field theory have long been investigated [4]. However, they have not often been applied to condensed matter physics, quantum optics or quantum communication. As an exceptional example, a fundamental lower bound of actuating energy for photon switching has recently been derived for input waves with negative energy density by a gedanken experiment [5].

In this paper, we present a protocol for near-critical two-level spin chains with nondegenerate ground states and large correlation lengths. In the analysis we assume that the number of spins is quite large, but finite. However, the results obtained in this paper can also be applied to the case of infinite degrees of freedom as long as an infinite limit of the number of spins is justified. We also concentrate on short time scales in which dynamical evolution induced by the Hamiltonian $H$ is negligible. We denote the difference between the largest eigenvalue and the smallest eigenvalue of $H$ by $\Delta E$. The timescale $t$ considered is assumed to satisfy

$$
t \ll \frac{1}{\Delta E} .
$$

Assuming this condition, it is valid to treat the time evolution operator as $\exp [-i t H] \sim I$. It should also be noted that the condition in eq. (1) can be weakened if a finite amount of energy $E_{\text {in }}$ less than $\Delta E$ is input to the spin chain by the energy sender as follows:

$$
t \ll \frac{1}{E_{\text {in }}} .
$$


On the other hand, we also assume that LOCC for the spins can be repeated many times even in a short time interval. Taking the site number difference between the two parties in the protocol as $\Delta n$ and the lattice spacing between nearest-neighbor sites as $a$, the time scale condition for many-round LOCC is expressed as

$$
t \gg a \Delta n / c,
$$

where $c$ is the light velocity. By taking the nonrelativistic limit $c \rightarrow \infty$, the relation in eq. (3) always holds. The proposed protocol is presented for one-dimensional qubit chain systems. Extending the protocol to spin chain systems with larger spins and larger dimensions is a straightforward task.

The paper is organized as follows. In section 2, we show that negative localized energy density naturally appears in the systems under study. In section 3, local energy conservation of the spin chain systems is discussed. In section 4, we discuss the measurement of local observables for the ground state, which is an important consideration for the QET protocol. In section 5, we propose the QET protocol. We conclude the paper in section 6 .

\section{Ground-State Entanglement and Negative Energy Density}

Let us consider a spin chain with a nondegenerate ground state. The Hamiltonian is given by a sum of semi-local components $T_{n}$ :

$$
H=\sum_{n} T_{n}
$$

Here $T_{n}$ are Hermitian operators given by

$$
T_{n}=\sum_{\gamma} \prod_{m=n-L}^{n+L} O_{m}^{(n, \gamma)},
$$


where $O_{m}^{(n, \gamma)}$ is a local Hermitian operator at site $m$ and the integer $L$ denotes the interaction range. If we take $L=1$, the nearest neighbor interaction can be treated. For example, the interaction of the Ising model with a transverse magnetic field satisfies $L=1$ and has $T_{n}$ such that

$$
T_{n}=-b \sigma_{n}^{z}-\frac{h}{2} \sigma_{n}^{x}\left(\sigma_{n+1}^{x}+\sigma_{n-1}^{x}\right)-\epsilon,
$$

where $b, h$ and $\epsilon$ are real constants. $T_{n}$ of the example has three terms in the right-hand side in eq. (5) with $\gamma=1,2,3$. These operators are given by

$$
\begin{aligned}
O_{n-1}^{(n, 1)} & =I, \\
O_{n}^{(n, 1)} & =-b \sigma_{n}^{z}-\epsilon, \\
O_{n+1}^{(n, 1)} & =I, \\
O_{n-1}^{(n, 2)} & =I, \\
O_{n}^{(n, 2)} & =-\frac{h}{2} \sigma_{n}^{x}, \\
O_{n+1}^{(n, 2)} & =\sigma_{n+1}^{x}, \\
O_{n-1}^{(n, 3)} & =\sigma_{n-1}^{x}, \\
O_{n}^{(n, 3)} & =-\frac{h}{2} \sigma_{n}^{x}, \\
O_{n+1}^{(n, 3)} & =I .
\end{aligned}
$$

$T_{n}$ describes the local energy density at site $n$. The ground state $|g\rangle$ is an eigenstate for the lowest eigenvalue $E_{0}$ of $H$. However, it is not guaranteed in general that $|g\rangle$ is an eigenstate of $T_{n}$. Without changing the dynamics at all, it is always possible to shift $T_{n}$ by adding constants. Hence, we are able to redefine $T_{n}$ to satisfy

$$
\left\langle g\left|T_{n}\right| g\right\rangle=0 .
$$

Then, the shifted value of $E_{0}$ becomes zero as follows:

$$
E_{0}=\langle g|H| g\rangle=\sum_{n}\left\langle g\left|T_{n}\right| g\right\rangle=0 .
$$

From the above, it is satisfied that

$$
H|g\rangle=0
$$


without any loss of generality. By this redefinition, $H$ becomes a non-negative operator and satisfies

$$
\operatorname{Tr}[\rho H] \geq 0
$$

for an arbitrary quantum state $\rho$. This choice of the energy origin simplifies the spin chain analysis, because we will often consider the energy difference between an excited state and the ground state.

If each $T_{n}$ is an exact local operator at site $n$, all $T_{n}$ can be simultaneously diagonalized and the ground state $|g\rangle$ is separable and an eigenstate for the lowest eigenvalue of each $T_{n}$. In such a situation, $T_{n}$ becomes non-negative. However, the condition is not sustained in general for cases with interactions between spins, and entangled ground states often appear. In the later discussion, we are interested in spin-chain models with entangled ground states. Let us consider an ordinary case of a correlation function $\left\langle g\left|T_{n} O_{m}\right| g\right\rangle$, not decomposed into $\left\langle g\left|T_{n}\right| g\right\rangle\left\langle g\left|O_{m}\right| g\right\rangle$ :

$$
\left\langle g\left|T_{n} O_{m}\right| g\right\rangle \neq\left\langle g\left|T_{n}\right| g\right\rangle\left\langle g\left|O_{m}\right| g\right\rangle
$$

for a certain site $n$ and local operator $O_{m}$ at site $m$ with $|n-m| \geq L+1$. Clearly, the state $|g\rangle$ is entangled because all separable ground states satisfy $\left\langle g\left|T_{n} O_{m}\right| g\right\rangle=\left\langle g\left|T_{n}\right| g\right\rangle\left\langle g\left|O_{m}\right| g\right\rangle$. It is proven easily from eq. (10) that the state $|g\rangle$ is not an eigenstate of $T_{n}$. The reason is as follows. If $T_{n}|g\rangle=c_{n}|g\rangle$ is satisfied for a certain real constant $c_{n}$, the correlation function must be written as

$$
\left\langle g\left|T_{n} O_{m}\right| g\right\rangle=c_{n}\left\langle g\left|O_{m}\right| g\right\rangle=\left\langle g\left|T_{n}\right| g\right\rangle\left\langle g\left|O_{m}\right| g\right\rangle,
$$

which contradicts eq. (10). Therefore we obtain the relation:

$$
T_{n}|g\rangle \neq c_{n}|g\rangle
$$

for arbitrary real constant $c_{n}$. eq. (11) gives important information about the emergence of a negative energy density as follows. Because the operator $T_{n}$ is a Hermitian operator acting on the total Hilbert space of the spin chain, $T_{n}$ can be spectrally decomposed into

$$
T_{n}=\sum_{\nu, k_{\nu}} \epsilon_{\nu}(n)\left|\epsilon_{\nu}(n), k_{\nu}, n\right\rangle\left\langle\epsilon_{\nu}(n), k_{\nu}, n\right|,
$$

where $\epsilon_{\nu}(n)$ are eigenvalues of $T_{n},\left|\epsilon_{\nu}(n), k_{\nu}, n\right\rangle$ are corresponding eigenstates and the index $k_{\nu}$ denotes the degeneracy freedom of the eigenvalue $\epsilon_{\nu}(n)$. Because $\left\{\left|\epsilon_{\nu}(n), k_{\nu}, n\right\rangle\right\}$ is a complete orthogonal set of basis state vectors of 
the total Hilbert space, the ground state in the total Hilbert space can be uniquely expanded as

$$
|g\rangle=\sum_{\nu, k_{\nu}} g_{\nu, k_{\nu}}(n)\left|\epsilon_{\nu}(n), k_{\nu}, n\right\rangle
$$

Using this expansion, eq. (7) gives

$$
\left\langle g\left|T_{n}\right| g\right\rangle=\sum_{\nu, k_{\nu}} \epsilon_{\nu}(n)\left|g_{\nu, k_{\nu}}(n)\right|^{2}=0 .
$$

Clearly, eq. (13) has no solutions if the lowest eigenvalue $\epsilon_{-}(n)$ of $T_{n}$ is positive. For the case of $\epsilon_{-}(n)=0$, eq. (13) has a solution with $g_{-, k_{-}}(n) \neq 0$. The other components $g_{\nu, k_{\nu}}(n)$ vanish. However, this solution implies that $T_{n}|g\rangle=c_{n}|g\rangle$ with $c_{n}=\epsilon_{-}(n)$ because of eq. (12) and apparently contradicts eq. (11). Therefore $\epsilon_{-}(n)$ does not take a zero value. Hence, it is concluded that $\epsilon_{-}(n)$ must be negative:

$$
\epsilon_{-}(n)=-\left|\epsilon_{-}(n)\right|<0 .
$$

The average energy density for the corresponding eigenstate $\left|\epsilon_{-}(n), k_{-}, n\right\rangle$ also becomes negative. It is thereby verified that there exist quantum states with negative energy density for spin chains satisfying eq. (10). It should be stressed again that even if a state has negative energy density over a certain region, there exists positive energy density at other regions and the total energy is not negative because of the non-negativity of $H$.

\section{Local Energy Conservation}

In this section, the local energy conservation of the spin chain system $S$ is explained. First, a free system, which is not coupled with external systems, is considered. The system evolves by the Hamiltonian in eq. (44). A connected site region is introduced, $V$, given by $\left[n_{i}, n_{f}\right]$ that satisfies $n_{f}-n_{i} \geq 2 L-1$. The energy of $V$ is defined by

$$
H_{V}=\sum_{n=n_{i}}^{n_{f}} T_{n} .
$$


In a free system, the Heisenberg operator of a Schrödinger operator $O$ is defined by $O^{(H)}(t)=e^{i t H} O e^{-i t H}$. The Heisenberg operator of $H_{V}$ evolves by the following equation:

$$
\frac{d}{d t} H_{V}^{(H)}=i\left[H, H_{V}^{(H)}\right]
$$

By substituting a relation given by

$$
H=\sum_{n \leq n_{i}-2 L-1} T_{n}^{(H)}+\sum_{n=n_{i}-2 L}^{n_{i}-1} T_{n}^{(H)}+H_{V}^{(H)}+\sum_{n=n_{f}+1}^{n_{f}+2 L} T_{n}^{(H)}+\sum_{n \geq n_{f}+2 L+1} T_{n}^{(H)}
$$

into eq. (15), it is possible to obtain

$$
\frac{d}{d t} H_{V}^{(H)}=i\left[\sum_{n=n_{i}-2 L}^{n_{i}-1} T_{n}^{(H)}+\sum_{n=n_{f}+1}^{n_{f}+2 L} T_{n}^{(H)}, H_{V}^{(H)}\right] .
$$

Substituting eq. (14) into the above equation yields the following energy conservation relation for an arbitrary initial state $|\psi\rangle$.

$$
\frac{d}{d t}\left\langle\psi\left|H_{V}^{(H)}\right| \psi\right\rangle=J_{n_{i}-1}-J_{n_{f}}
$$

where the above energy fluxes are given by

$$
J_{n}=i\left\langle\psi\left|\left[\sum_{m=n-2 L+1}^{n} T_{m}^{(H)}, \sum_{m^{\prime}=n+1}^{n+2 L} T_{m^{\prime}}^{(H)}\right]\right| \psi\right\rangle
$$

If $J_{n_{i}-1}=J_{n_{f}}=0$, the energy of $V$ does not change at all.

Next, a situation is considered in which the spin chain $S$ is locally coupled with external systems $D$ and $C$. $S$ interacts with the external systems only at site $n_{o}$. The coupling can be switched on and off effectively by the time evolution of the switch system $C$. System $D$ locally controls the spin chain in the switch-on interval. The free part of the total Hamiltonian is then written as

$$
H_{o}=H+H_{C}+H_{D}
$$


where $H_{C}$ is the free Hamiltonian of $C$, and $H_{D}$ is of $D$. In order to capture the essence of physics, example cases are considered in which the switch system $C$ is a one-dimensional bosonic Schrödinger field $\Psi(x)$ with Hamiltonian

$$
\begin{aligned}
H_{C}= & -\frac{i v}{2} \int_{-\infty}^{\infty}\left[\Psi^{\dagger}(x) \partial_{x} \Psi(x)-\partial_{x} \Psi^{\dagger}(x) \Psi(x)\right] d x \\
& +\frac{1}{2 M} \int_{-\infty}^{\infty} \partial_{x} \Psi^{\dagger}(x) \partial_{x} \Psi(x) d x
\end{aligned}
$$

where $v$ and $M$ are positive real paramters and the operators $\Psi(x)$ and $\Psi^{\dagger}(x)$ satisfy

$$
\begin{aligned}
{\left[\Psi(x), \Psi^{\dagger}\left(x^{\prime}\right)\right] } & =i \delta\left(x-x^{\prime}\right), \\
{\left[\Psi(x), \Psi\left(x^{\prime}\right)\right] } & =0, \\
{\left[\Psi^{\dagger}(x), \Psi^{\dagger}\left(x^{\prime}\right)\right] } & =0 .
\end{aligned}
$$

In later discussion, we take the mass parameter $M$ very large. Then $v$ bocomes the propagating velocity of right-moving excitation of the field outside the interaction region:

$$
e^{i t H_{o}} \Psi^{\dagger}(x) e^{-i t H_{o}} \approx \Psi^{\dagger}(x-v t) .
$$

The vacuum state $|0\rangle$ of $\Psi$ is defined by $\Psi(x)|0\rangle=0$. By use of the explicit example of $C$, a model of local operation for the spin $S$ at site $n_{o}$ with energy conservation can be made. The total Hamiltonian is given by

$$
\begin{aligned}
H_{t o t} & =H_{o}+H_{i n t}^{\left(n_{o}\right)} \\
H_{\text {int }}^{\left(n_{o}\right)} & =g K_{S+D}^{\left(n_{o}\right)} \int_{-d}^{d} \Psi^{\dagger}(x) \Psi(x) d x
\end{aligned}
$$

Here $g$ is a real coupling constant, $d$ fixes the interaction region of $\Psi$ to $S$ and $D . K_{S+D}^{\left(n_{o}\right)}$ is a Hermitian operator acting on the Hilbert space of the composite system $S$ and $D$, and can be decomposed as

$$
K_{S+D}^{\left(n_{o}\right)}=I_{n_{o}} O_{D}+\vec{\sigma}_{n_{o}} \cdot \vec{O}_{D}
$$

where $O_{D}$ and $\vec{O}_{D}$ are Hermitian operators acting on the Hilbert space of $D$. In this model, energy conservation is satisfied via 


$$
\frac{d}{d t}\left(\hat{H}(t)+\hat{H}_{C}(t)+\hat{H}_{D}(t)+\hat{H}_{i n t}^{\left(n_{o}\right)}(t)\right)=0,
$$

where the hat operators denote the Heisenberg operator corresponding to Schrödinger operators:

$$
\hat{O}(t)=e^{i t H_{t o t}} O e^{-i t H_{t o t}} .
$$

Switching is realized by scattering a wave packet of $\Psi$ via the interaction in eq. (17). Let us set the initial state of $C$ to a coherent state

$$
\left|\phi_{C}\right\rangle=N_{\phi} \exp \left(\int_{x_{0}}^{x_{1}} \phi(x) \Psi^{\dagger}(x) d x\right)|0\rangle,
$$

where $\phi(x)$ is amplitude of the coherent state and localized in the space region $\left[x_{0}, x_{1}\right]$ such that $x_{1}<-d$. Let us assume that the total initial state is a product state given by $|I\rangle=\left|\psi_{S}\right\rangle\left|\psi_{D}\right\rangle\left|\phi_{C}\right\rangle$, where $\left|\psi_{S}\right\rangle$ is the initial state of $S$ and $\left|\psi_{D}\right\rangle$ is the initial state of $D$. It is noted that in the initial phase, the interaction in eq. (17) is switched off because no wave of $\Psi$ exists inside the interaction region $[-d, d]$ and $H_{i n t}^{\left(n_{o}\right)}$ has no contribution. The wave packet evolves freely with velocity $v$ until a part of the packet reaches $[-d, d]$. When a part of the wave packet stays in $[-d, d]$, the interaction in eq. (17) is effectively switched on and energy can be exchanged among $S, C$ and $D$. In the interval, the wave packet of $\Psi$ is scattered by the interaction. After the scattering process, the scattered wave of $\Psi$ escapes with velocity $v$ from the interaction region $[-d, d]$ and $H_{i n t}^{\left(n_{o}\right)}$ again has no contribution. Hence, taking account of the disappearance of $H_{i n t}^{\left(n_{o}\right)}$ contribution in the past and future of the scattering events, energy conservation in eq. (18) clearly yields the following relation:

$$
\left\langle I\left|\left(\hat{H}\left(t_{i}\right)+\hat{H}_{C}\left(t_{i}\right)+\hat{H}_{D}\left(t_{i}\right)\right)\right| I\right\rangle=\left\langle I\left|\left(\hat{H}\left(t_{f}\right)+\hat{H}_{C}\left(t_{f}\right)+\hat{H}_{D}\left(t_{f}\right)\right)\right| I\right\rangle,
$$

where $t_{i}$ is the initial time, and $t_{f}$ is the final time of the scattering process. It should be stressed that the scattering interval $t_{f}-t_{i}$ can go to zero as $v$ and $g$ approach infinity. It should be recalled that the nonrelativistic limit is assumed in this paper, and the speed of light is assumed to be infinity. Thus, $v$ can also approach infinity. At this limit, the time evolution of the spins of $S$ at all sites except site $n_{o}$ can be neglected during the short-time scattering 
process. Then, energy exchange occurs not globally but locally among spins around site $n_{o}, C$ and $D$. Therefore, eq. (19) can be rewritten as

$$
\begin{aligned}
& \left\langle I\left|\left(\sum_{n=n_{o}-L}^{n_{o}+L} \hat{T}_{n}\left(t_{i}\right)+\hat{H}_{C}\left(t_{i}\right)+\hat{H}_{D}\left(t_{i}\right)\right)\right| I\right\rangle \\
= & \left\langle I\left|\left(\sum_{n=n_{o}-L}^{n_{o}+L} \hat{T}_{n}\left(t_{f}\right)+\hat{H}_{C}\left(t_{f}\right)+\hat{H}_{D}\left(t_{f}\right)\right)\right| I\right\rangle .
\end{aligned}
$$

If $\left\langle I\left|\left(\hat{H}\left(t_{f}\right)-\hat{H}\left(t_{i}\right)\right)\right| I\right\rangle$ is positive, the spin chain gets energy from $C$ and $D$ in the short-time scattering interval. Inversely, if $\left\langle I\left|\left(\hat{H}\left(t_{f}\right)-\hat{H}\left(t_{i}\right)\right)\right| I\right\rangle$ is negative, the spin chain gives energy to the composite system of $C$ and $D$. This fact becomes significant in the QET protocol proposed later.

\section{Local Measurement of the Ground State and Entanglement Breaking}

For the QET protocol, it is important to consider the local measurement of an entangled ground state $|g\rangle$ of a spin chain. Let us consider a spin at site $n_{A}$ in the spin chain and a Hermitian unitary local operator $\sigma_{A}=\vec{u}_{A} \cdot \vec{\sigma}_{n_{A}}$. Here $\vec{u}_{A}$ is a three-dimensional real unit vector and $\vec{\sigma}_{n_{A}}$ is the Pauli spin matrix vector at site $n_{A}$. The eigenvalues of $\sigma_{A}$ are $(-1)^{\mu}$ with $\mu=0,1$. The spectral expansion of $\sigma_{A}$ is given by

$$
\sigma_{A}=\vec{u}_{A} \cdot \vec{\sigma}_{A}=\sum_{\mu=0,1}(-1)^{\mu} P_{A}(\mu)
$$

where $P_{A}(\mu)$ is a projective operator onto the eigensubspace with $\mu$. Consider that Alice at site $n_{A}$ performs a projective measurement of $\sigma_{A}$ for the ground state $|g\rangle$ and assume that she obtains the measurement result $\mu$. The postmeasurement state with $\mu$ is given by

$$
\frac{1}{\sqrt{p_{A}(\mu)}} P_{A}(\mu)|g\rangle,
$$

where $p_{A}(\mu)=\left\langle g\left|P_{A}(\mu)\right| g\right\rangle$. Therefore, the average post-measurement state is a mixed quantum state $\rho^{\prime}$ given by 


$$
\begin{aligned}
\rho^{\prime} & =\sum_{\mu=0,1} p_{A}(\mu) \frac{1}{\sqrt{p_{A}(\mu)}} P_{A}(\mu)|g\rangle\langle g| P_{A}(\mu) \frac{1}{\sqrt{p_{A}(\mu)}} \\
& =\sum_{\mu=0,1} P_{A}(\mu)|g\rangle\langle g| P_{A}(\mu) .
\end{aligned}
$$

It should be noted that $\rho^{\prime}$ is a quantum state which satisfies

$$
\operatorname{Tr}_{n_{A}}\left[\rho^{\prime}\right]=\operatorname{Tr}_{n_{A}}[|g\rangle\langle g|],
$$

where $\operatorname{Tr}_{n_{A}}$ means a partial trace in terms of the measured spin at site $n_{A}$. Therefore, the quantum fluctuation of $\rho^{\prime}$ is the same as that of the ground state except at site $n_{A}$. It should also be noted that the above projective measurement needs energy. Because the ground state has zero energy, the input $E_{A}$ is calculated as follows:

$$
E_{A}=\operatorname{Tr}\left[\rho^{\prime} H\right]-\langle g|H| g\rangle=\sum_{\mu=0,1}\left\langle g\left|P_{A}(\mu) H P_{A}(\mu)\right| g\right\rangle .
$$

Because of the non-negativity of $H, E_{A}$ is non-negative. In general, $P_{A}(\mu)|g\rangle$ is not proportional to $|g\rangle$ due to entanglement and we obtain a positive value of $E_{A}$. Because the measurement is performed locally, the excitation energy of the spin chain is localized around site $n_{A}$ soon after the measurement. For convenience, we introduce a localized energy operator around site $n$ by

$$
H_{n}=\sum_{m=n-L}^{n+L} T_{m} .
$$

$H_{n}$ includes all $T_{m}$ 's for which expectation values change if quantum operations act on site $n$. From eq. (25), it can be shown that

$$
\operatorname{Tr}\left[\rho^{\prime} H_{n_{A}}\right]=E_{A} .
$$

Also, it is proven that

$$
\operatorname{Tr}\left[\rho^{\prime} T_{n}\right]=0
$$

where $\left|n-n_{A}\right|>L$. eq. (26) implies localization of the energy distribution around site $n_{A}$. 
Next, consider that Alice at site $n_{A}$ attempts to completely withdraw the input energy $E_{A}$ by local operations soon after the measurement. Her available processes are expressed by arbitrary trace-preserving completely-positive (TPCP) maps $\Gamma_{A}$ of the spin state at site $n_{A}$. In spite of any use of local TPCP maps, her attempt cannot be achieved because the local measurement breaks the entanglement between the spin at site $n_{A}$ and different-site spins in the spin chain. If Alice wants to recover the original ground state, she must recreate the entanglement broken by the first measurement. However, entanglement generation needs nonlocal operations in general. Therefore, she cannot recover the ground state perfectly by her local operations alone. This observation implies that there is nonvanishing residual energy $E_{\text {res }}$ in Alice's local cooling process to extract the input energy:

$$
E_{\text {res }}=\min _{\Gamma_{A}}\left(\operatorname{Tr}\left[\Gamma_{A}\left[\rho^{\prime}\right] H_{n_{A}}\right]\right)>0 .
$$

Therefore, interestingly, Alice is not able to use the residual energy even though it is right in front of her. In the next section, we argue that a part of the residual energy can be extracted by Bob, who is situated apart from Alice and performs local operations dependent on the measurement result $\mu$ of $\sigma_{A}$ to a spin on the chain in front of him. This protocol is QET.

\section{Quantum Energy Teleportation}

In this section, we discuss QET for spin chain systems. As mentioned in the introduction, we concentrate on short time scales, in which dynamical evolution induced by the Hamiltonian of the spin chain is negligible. On the other hand, we also assume the nonrelativistic limit that LOCC for the spins can be repeated many times even in a short time interval. Initially, Alice and Bob share many near-critical spin chains in the ground state $|g\rangle$, which is entangled and has a large correlation length $l$. Alice is situated at site $n_{A}$ and Bob at site $n_{B}$. Alice is a good distance from Bob: $\left|n_{A}-n_{B}\right| \sim O(l) \gg 1$. Consider a protocol consisting of the following steps. (I) Alice performs a measurement of a local observable of the spin at site $n_{A}$ and obtains the measurement result $\mu$. In the measurement process, the spin chain is locally 
excited with some energy input. (II) Alice announces to Bob the result $\mu$ by a classical channel at the speed of light or near the light velocity. Because the time interval of the annoucement is very short, the system does not evolve and no energy flow appears between Alice and Bob. (III) Bob performs a local operation dependent on $\mu$ to the spin at site $n_{B}$. QET is defined by this protocol if the expectation value of $H_{n_{B}}$ is negative after the local operation of Bob. Because the expectation value of $H_{n_{B}}$ is zero just before Bob's operation and local energy conservation holds around Bob's region as mentioned in section 3, the negative value after Bob's operation implies that energy release occurs around site $n_{B}$ to external systems during the operation interval. (For the case in section 3, the energy moves from the spin chain $S$ to the external systems $C$ and $D$.) If Alice inputs no energy into the spin chain or Bob does not use the information about $\mu$, Bob is not able to extract energy from the spin chain at all. Therefore, it can be said that a part of the energy input by Alice's measurement at site $n_{A}$ is effectively transported to site $n_{B}$ by LOCC. If we select as the initial state of the spin chain, not the ground state but an excited state which has nonzero energy distribution around Bob, it is not unusual that Bob's local operation can extract energy from the spin chain. However, in QET, the ground state is actually selected as the initial state and the local energy extraction is nontrivial.

Next we propose an explicit protocol of QET. The protocol with $L=1$ is illustrated in Figure 1.

(I) Alice performs a projective measurement of the observable $\sigma_{A}$ in eq. (21) for the ground state $|g\rangle$ and obtains the measurement result $\mu=0$ or 1. Alice inputs energy $E_{A}$ in eq. (24) to the spin chain system in order to achieve the local measurement, as seen in section 4 .

(II)Alice announces to Bob the result $\mu$ by a classical channel. In the announcement process, both time evolution of the system and emergence of energy flux do not happen because the time interval is assumed very short.

(III) To a spin at site $n_{B}$, Bob performs a local unitary operation $V_{B}(\mu)$ depending on the value of $\mu$, which is defined by

$$
V_{B}(\mu)=I \cos \theta+i(-1)^{\mu} \sigma_{B} \sin \theta .
$$


Here $\sigma_{B}=\vec{u}_{B} \cdot \vec{\sigma}_{n_{B}}, \vec{u}_{B}$ is a three-dimensional real unit vector and $\vec{\sigma}_{n_{B}}$ is the Pauli spin matrix vector at site $n_{B}$. The real parameter $\theta$ is defined by

$$
\begin{aligned}
\cos (2 \theta) & =\frac{\xi}{\sqrt{\xi^{2}+\eta^{2}}}, \\
\sin (2 \theta) & =-\frac{\eta}{\sqrt{\xi^{2}+\eta^{2}}} .
\end{aligned}
$$

where the real parameters $\xi$ and $\eta$ are given by

$$
\begin{gathered}
\xi=\left\langle g\left|\sigma_{B} H \sigma_{B}\right| g\right\rangle, \\
\eta=\left\langle g\left|\sigma_{A} \dot{\sigma}_{B}\right| g\right\rangle
\end{gathered}
$$

with

$$
\dot{\sigma}_{B}=i\left[H_{n_{B}}, \sigma_{B}\right] .
$$

For the case with $\eta \neq 0$, Bob obtains positive energy:

$$
E_{B}=\frac{1}{2}\left[\sqrt{\xi^{2}+\eta^{2}}-\xi\right]
$$

on average from the spin chain in the process of the local operation.

The value of $\xi$ is non-negative due to the non-negativity of $H$. Because of the hermicity and commutativity of $\sigma_{A}$ and $\dot{\sigma}_{B}$, the reality of $\eta$ is easily proven as follows:

$$
\begin{aligned}
\eta^{*} & =\left\langle g\left|\sigma_{A} \dot{\sigma}_{B}\right| g\right\rangle^{*}=\left\langle g\left|\dot{\sigma}_{B} \sigma_{A}\right| g\right\rangle \\
& =\left\langle g\left|\sigma_{A} \dot{\sigma}_{B}\right| g\right\rangle=\eta .
\end{aligned}
$$

Using $\left[T_{n}, \sigma_{B}\right]=0$ for $\left|n-n_{B}\right|>L$, eq. (32) can be rewritten as

$$
\dot{\sigma}_{B}=i\left[H, \sigma_{B}\right] \text {, }
$$

and thus $\dot{\sigma}_{B}$ can be interpreted as a time-derivative operator of the Heisenberg operator $\exp [i t H] \sigma_{B} \exp [-i t H]$ at $t=0$. After Bob's operation, the post-measurement state in eq. (22) is transformed into

$$
\frac{1}{\sqrt{p_{A}(\mu)}} V_{B}(\mu) P_{A}(\mu)|g\rangle \text {. }
$$


Here we have neglected time evolution of the spin chain between Alice's measurement and Bob's operation assuming the relation of eq. (1) or eq. (2). The average state is given by

$$
\begin{aligned}
\rho & =\sum_{\mu=0,1} p_{A}(\mu) \frac{1}{\sqrt{p_{A}(\mu)}} V_{B}(\mu) P_{A}(\mu)|g\rangle\langle g| P_{A}(\mu) V_{B}^{\dagger}(\mu) \frac{1}{\sqrt{p_{A}(\mu)}} \\
& =\sum_{\mu=0,1} V_{B}(\mu) P_{A}(\mu)|g\rangle\langle g| P_{A}(\mu) V_{B}^{\dagger}(\mu) .
\end{aligned}
$$

It is straightforward to calculate the average localized energy $\operatorname{Tr}\left[\rho H_{n_{B}}\right]$ after Bob's operation, as follows. Firstly, by the commutativity of $P_{A}(\mu)$ and $V_{B}^{\dagger}(\mu) H_{n_{B}} V_{B}(\mu)$, we obtain

$$
\begin{aligned}
\operatorname{Tr}\left[\rho H_{n_{B}}\right] & =\sum_{\mu=0,1}\left\langle g\left|P_{A}(\mu)\left(V_{B}^{\dagger}(\mu) H_{n_{B}} V_{B}(\mu)\right) P_{A}(\mu)\right| g\right\rangle \\
& =\sum_{\mu=0,1}\left\langle g\left|P_{A}(\mu)\left(V_{B}^{\dagger}(\mu) H_{n_{B}} V_{B}(\mu)\right)\right| g\right\rangle,
\end{aligned}
$$

where we have used $P_{A}(\mu)^{2}=P_{A}(\mu)$. Substituting eq. (27) into the above relation yields the following expression of $\operatorname{Tr}\left[\rho H_{n_{B}}\right]$ :

$$
\begin{aligned}
& \operatorname{Tr}\left[\rho H_{n_{B}}\right] \\
= & \sum_{\mu=0,1}\left\langle g\left|P_{A}(\mu)\left(I \cos \theta-i(-1)^{\mu} \sigma_{B} \sin \theta\right) H_{n_{B}}\left(I \cos \theta+i(-1)^{\mu} \sigma_{B} \sin \theta\right)\right| g\right\rangle \\
= & \cos ^{2} \theta\left\langle g\left|\left(\sum_{\mu=0,1} P_{A}(\mu)\right) H_{n_{B}}\right| g\right\rangle+\sin ^{2} \theta\left\langle g\left|\left(\sum_{\mu=0,1} P_{A}(\mu)\right) \sigma_{B} H_{n_{B}} \sigma_{B}\right| g\right\rangle \\
& +i \cos \theta \sin \theta\left\langle g\left|\left(\sum_{\mu=0,1}(-1)^{\mu} P_{A}(\mu)\right)\left[H_{n_{B}}, \sigma_{B}\right]\right| g\right\rangle .
\end{aligned}
$$

By taking account of the completeness relation of $P_{A}(\mu)$ and the spectral decomposition of $\sigma_{A}$ in eq. (21), we get the following relation:

$$
\begin{aligned}
\operatorname{Tr}\left[\rho H_{n_{B}}\right]= & \cos ^{2} \theta\left\langle g\left|H_{n_{B}}\right| g\right\rangle+\sin ^{2} \theta\left\langle g\left|\sigma_{B} H_{n_{B}} \sigma_{B}\right| g\right\rangle \\
& +i \cos \theta \sin \theta\left\langle g\left|\sigma_{A}\left[H_{n_{B}}, \sigma_{B}\right]\right| g\right\rangle .
\end{aligned}
$$


Because $\left\langle g\left|H_{n_{B}}\right| g\right\rangle=0$ is satisfied, the form given by

$$
\operatorname{Tr}\left[\rho H_{n_{B}}\right]=\left\langle g\left|\sigma_{B} H_{n_{B}} \sigma_{B}\right| g\right\rangle \sin ^{2} \theta+\eta \cos \theta \sin \theta
$$

is obtained. Here, the relation:

$$
\begin{aligned}
\left\langle g\left|\sigma_{B} H_{n_{B}} \sigma_{B}\right| g\right\rangle & =\left\langle g\left|\sigma_{B}\left(H-\sum_{n^{\prime} \notin\left[n_{B}-L, n_{B}+L\right]} T_{n^{\prime}}\right) \sigma_{B}\right| g\right\rangle \\
& =\left\langle g\left|\sigma_{B} H \sigma_{B}\right| g\right\rangle=\xi
\end{aligned}
$$

is satisfied due to the following relation:

$$
\left\langle g\left|\sigma_{B} T_{n^{\prime}} \sigma_{B}\right| g\right\rangle=\left\langle g\left|\sigma_{B} \sigma_{B} T_{n^{\prime}}\right| g\right\rangle=\left\langle g\left|T_{n^{\prime}}\right| g\right\rangle=0
$$

for $n^{\prime} \notin\left[n_{B}-L, n_{B}+L\right]$. Thus, eq. (35) is rewritten as

$$
\begin{aligned}
\operatorname{Tr}\left[\rho H_{n_{B}}\right] & =\xi \sin ^{2} \theta+\eta \cos \theta \sin \theta \\
& =\frac{\xi}{2}(1-\cos (2 \theta))+\frac{\eta}{2} \sin (2 \theta) .
\end{aligned}
$$

The parameter $\theta$ is now fixed to make $\operatorname{Tr}\left[\rho H_{n_{B}}\right]$ as negative as possible. This can be achieved by taking $\theta$ as in eqs. (28) and (29). $\operatorname{Tr}\left[\rho H_{n_{B}}\right]$ is then evaluated as

$$
\operatorname{Tr}\left[\rho H_{n_{B}}\right]=\frac{1}{2}\left[\xi-\sqrt{\xi^{2}+\eta^{2}}\right]
$$

by substituting eqs. (28) and (29) into the relation derived from eq. (36). If $\eta \neq 0$, it is clear that $\operatorname{Tr}\left[\rho H_{n_{B}}\right]$ in eq. (37) is negative:

$$
\operatorname{Tr}\left[\rho H_{n_{B}}\right]<0 .
$$

eq. (38) is a significant result. Before step (III), the energy around Bob is zero:

$$
\operatorname{Tr}\left[\rho^{\prime} H_{n_{B}}\right]=0 .
$$

After Bob's local operations in step (III), the localized energy around site $n_{B}$ becomes negative. Respecting local energy conservation explained in section 3 , this means that positive energy $E_{B}$ must be emitted from the spin chain 
to Bob (more precisely, to Bob's devices to perform $V_{B}(\mu)$ ). More explicitly, local energy conservation around site $n_{B}$ yields the relation:

$$
E_{B}+\operatorname{Tr}\left[\rho H_{n_{B}}\right]=\operatorname{Tr}\left[\rho^{\prime} H_{n_{B}}\right]=0 .
$$

From this relation, we finally obtain eq. (33) as follows:

$$
\begin{aligned}
E_{B} & =\operatorname{Tr}\left[\rho^{\prime} H_{n_{B}}\right]-\operatorname{Tr}\left[\rho H_{n_{B}}\right] \\
& =\frac{1}{2}\left[\sqrt{\xi^{2}+\eta^{2}}-\xi\right]
\end{aligned}
$$

As seen in eq. (31), $\eta$ is given by a two-point correlation function of (semi)local operators. If the ground state is separable, it turns out from eq. (34) and $H|g\rangle=0$ that $\eta$ vanishes as follows:

$$
\begin{aligned}
\eta & =\left\langle g\left|\sigma_{A} \dot{\sigma}_{B}\right| g\right\rangle=\left\langle g\left|\sigma_{A}\right| g\right\rangle\left\langle g\left|\dot{\sigma}_{B}\right| g\right\rangle \\
& =i\left\langle g\left|\sigma_{A}\right| g\right\rangle\left\langle g\left|\left(H \sigma_{B}-\sigma_{B} H\right)\right| g\right\rangle=0 .
\end{aligned}
$$

Therefore, it can easily be checked from eq. (33) that Bob gains no energy, as should be the case. However, the correlation function $\eta$ does not vanish in general because entanglement yields correlations among the quantum fluctuations at each point. For example, the critical Ising spin chains with transversal magnetic field have nonvanishing $\eta[6]$. In general, any spin chain models without fine-tuning may have nonvanishing $\eta$, just like the Ising spin chains, and the protocol in this paper becomes effective barring fine-tuned exceptions. Schematic plots of the expectation values of energy density for the steps of the QET are given in Figs. 2-4. From these figures, it can be easily understood that there exists no energy flow between Alice and Bob. Energy is locally transported from the spin chain to Bob's device for the operation by simultaneously generating negative energy $-E_{B}$ in the spin chain and positive energy $+E_{B}$ in the device of Bob. Hence, local energy conservation is exactly maintained in the protocol.

It is worth commenting here that the ground state $|g\rangle$ has a typical correlation site length $l$, over which the correlation between two sites and the value of $\eta$ decay rapidly. Hence, the protocol is more effective in teleporting energy between Alice and Bob with $\left|n_{A}-n_{B}\right| \lesssim l$, because the energy gain of Bob increases when $\eta$ is large. To achieve long-range QET, it is preferable to choose near-critical spin chain systems which have entangled ground states with long-range correlation, that is, $l \gg 1$. For example, the near-critical 
Ising spin chains in the presence of transversal magnetic field with $b \sim h>0$ in eq. (6) may be good candidates.

In the above analysis, we have shown that Bob obtains energy from the spin chain. However, even after the last step of the protocol, there exists energy $E_{A}$ that Alice had to first deposit to the spin chain by her measurement. Actually, using $H_{n_{A}} V_{B}(\mu)=V_{B}(\mu) H_{n_{A}}$, we are able to check easily that $\operatorname{Tr}\left[\rho H_{n_{A}}\right]=\operatorname{Tr}\left[\rho^{\prime} H_{n_{A}}\right]$. Let us imagine that Alice attempts to completely withdraw $E_{A}$ by local operations after step (III). If this was possible, the energy gain $E_{B}$ of Bob might have no cost. However, if so, the total energy of the spin chain becomes equal to $-E_{B}$ and negative. Meanwhile, we know that the total energy of the spin chains must be nonnegative. Hence, Alice cannot withdraw energy larger than $E_{A}-E_{B}$ by local operations at site $n_{A}$. The reason of existence of the residual energy has been already explained in section 4 from the viewpoint of entanglement breaking. It may be instructive to stress the simple fact that if the deposited energy $E_{A}$ vanishes, $E_{B}$ also vanishes. This implies that Bob releases a part of energy poured by Alice's measurement.

\section{Conclusion}

In this paper, a protocol of QET is proposed in spin chain systems that respects all physical laws including causality and local energy conservation. Energy is effectively transported from Alice to Bob by only LOCC, using local excitations with negative energy and ground-state entanglement. The energy input by Alice is given by eq. (24) and the energy gain by Bob is given by eq. (33).

If we do not consider the ground state, but rather an excited state of the spin chain which has nonzero energy distribution around Bob, it is not anomalous that Bob can extract energy from the spin chain. In the QET protocol, the initial state is set to be the ground state and energy extraction by Bob is nontrivial. At first glance, energy extraction from the ground state by Bob appears impossible due to local energy conservation because Alice only excites locally the spin chain by her measurements. Based on this, we may wonder why energy is teleported in QET without any physical agent in the protocol. The answer may be briefly summarized as follows. As stressed in section 2, quantum mechanics allows negative-energy-density regions by 
controlling quantum fluctuation. Hence, even if Bob has no energy on average around him, the value of the energy density around him can decrease. Therefore, the ground state can be locally regarded as "an excited state", compared with the state with negative energy around Bob. In the QET protocol, Bob extracts this local excitation energy by using Alice's measurement result. In this way, it can be said that the energy, which Bob will obtain, existed around Bob before the start of the QET protocol. Therefore, we do not need any transfer of energy from Alice to Bob. Of course, the energy hidden behind Bob's region is not always available. In the protocol, classical information about the measurement result of Alice becomes a key to extract energy by Bob. Without knowledge about the measurement result, Bob cannot get any energy from the local ground state. The essential reason why the measurement result allows Bob to get energy is as follows. Because the spin of Alice is entangled with spins around Bob, the measurement result includes information about the quantum fluctuations around Bob. Bob infers from the measurement information how the quantum fluctuations behave around him and can choose a unitary operation to extract but not give energy from the spin chain. Consequently, the protocol is able to transport energy by LOCC without breaking local energy conservation and without the existence of any excited physical entity.

\section{Acknowledgments}

This research was partially supported by the SCOPE project of the MIC.

\section{References}

[1] C.H. Bennett, G. Brassard, C. Crépeau, R. Jozsa, A. Peres, and W.K. Wootters: Phys. Rev. Lett. 70 (1993) 1895.

[2] S. Bose: Phys. Rev. Lett. 91 (2003) 207901; F. Verstraete, M.A. Mart'mDelgado, and J.I. Cirac: Phys. Rev. Lett. 92 (2004) 087201.

[3] L. Amico, R. Fazio, A. Osterloh, and V. Vedral: quant-ph/0703044. 
[4] N.D. Birrell and P.C.W. Davies: Quantum Fields in Curved Space (Cambridge Univ. Press, 1982) p. 269.

[5] M. Hotta: Phys. Lett. A372 (2008) 3752.

[6] M. Hotta: Phys. Lett. A372(2008) 5671.

[7] P. Pfeuty: Ann. Phys. 57(1970)79. 


\section{Figure Caption}

\section{Figure 1: QET protocol}

(1) The eigenvalues of $\vec{u}_{A} \cdot \vec{\sigma}_{A}$ are $(-1)^{\mu}$ with $\mu=0,1$. Alice performs a local projective measurement of the observable $\vec{u}_{A} \cdot \vec{\sigma}_{A}$ to her spin in the ground state $|g\rangle$ and obtains the measurement result $\mu$. Alice must input energy $E_{A}$ to the spin chain system in order to achieve the local measurement. (2) Alice announces to Bob the result $\mu$ by a classical channel. (3) Bob performs a local unitary operation $V_{B}(\mu)$ to his spin, depending on the value of $\mu$. Bob obtains energy output $E_{B}$ on average from the spin chain system in the process of the local operation.

Figure 2: Schematic plot of the expectation value of energy density in Step (I). Alice measures $\vec{u}_{A} \cdot \vec{\sigma}_{A}$ and obtains the result $\mu$. The energy $E_{A}$ is poured into the spin chain by the local measurement.

Figure 3: Schematic plot of the expectation value of energy density in Step (II). Alice announces $\mu$ to Bob. Bob perfomes result-dependent unitary operation to his spin.

Figure 4: Schematic plot of the expectation value of energy density in Step (III). Negative energy $-E_{B}$ appears in the spin chain and positive energy $+E_{B}$ is released outside. Bob is able to use the energy. 


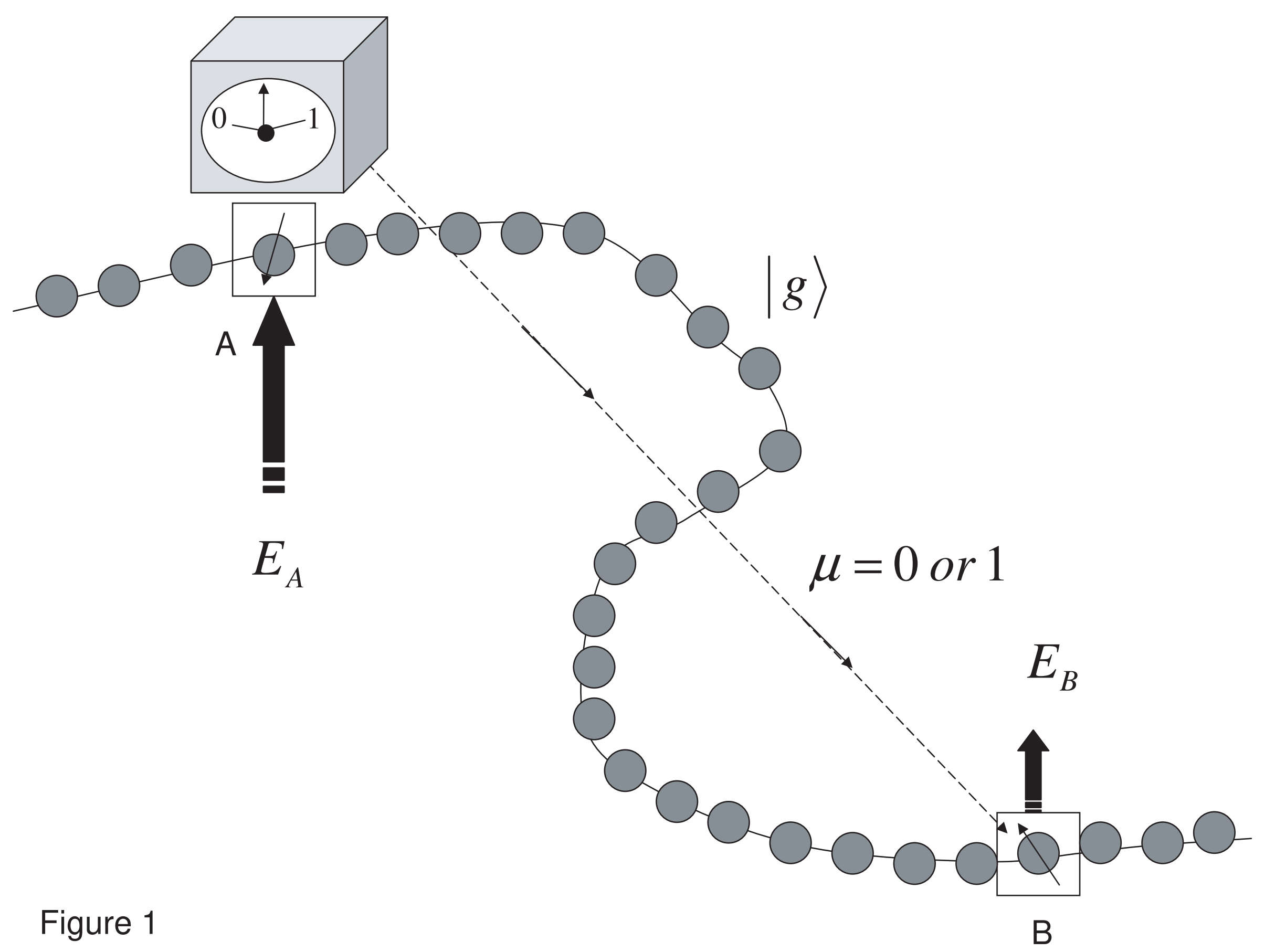




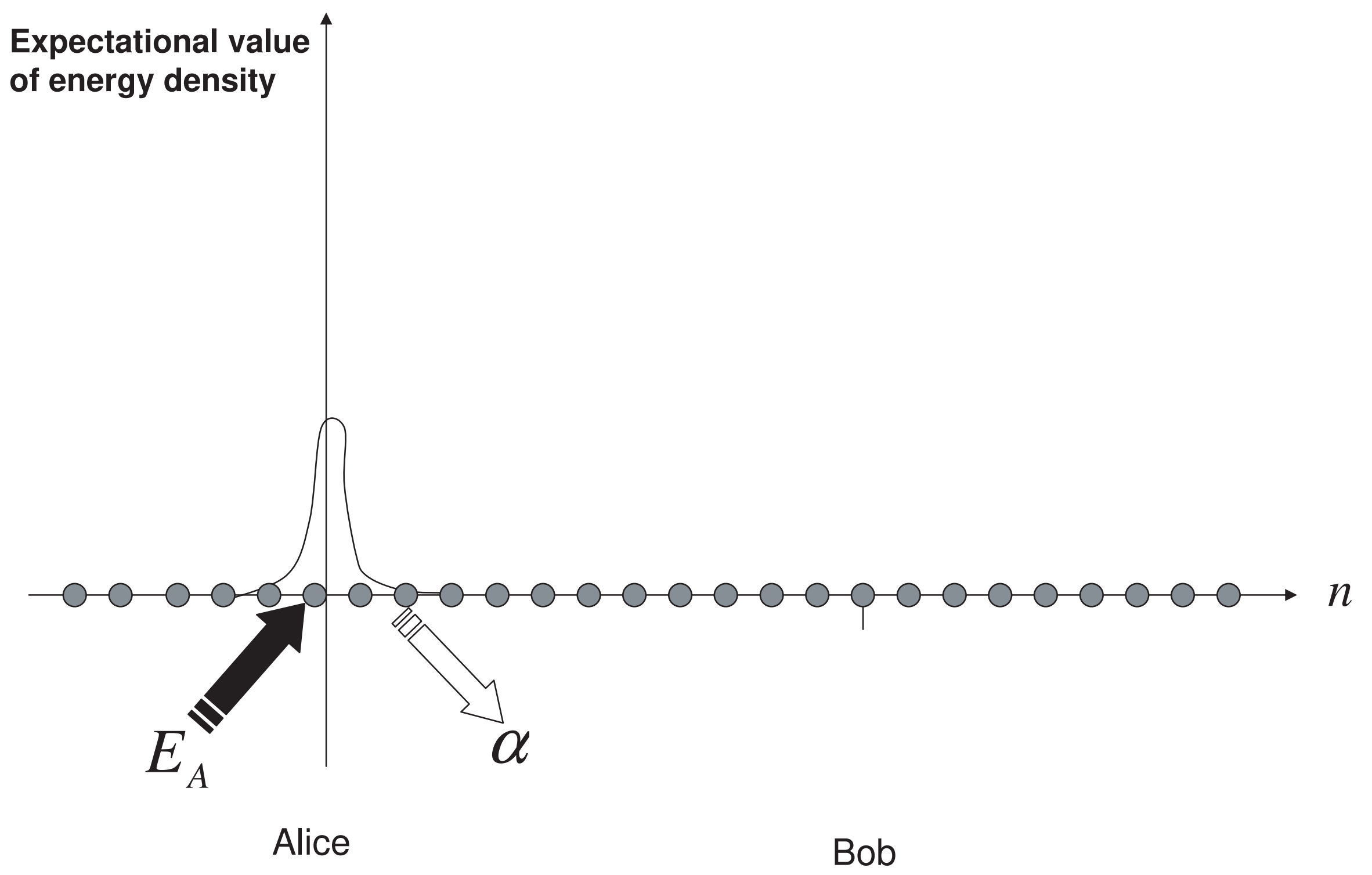

Figure 2 


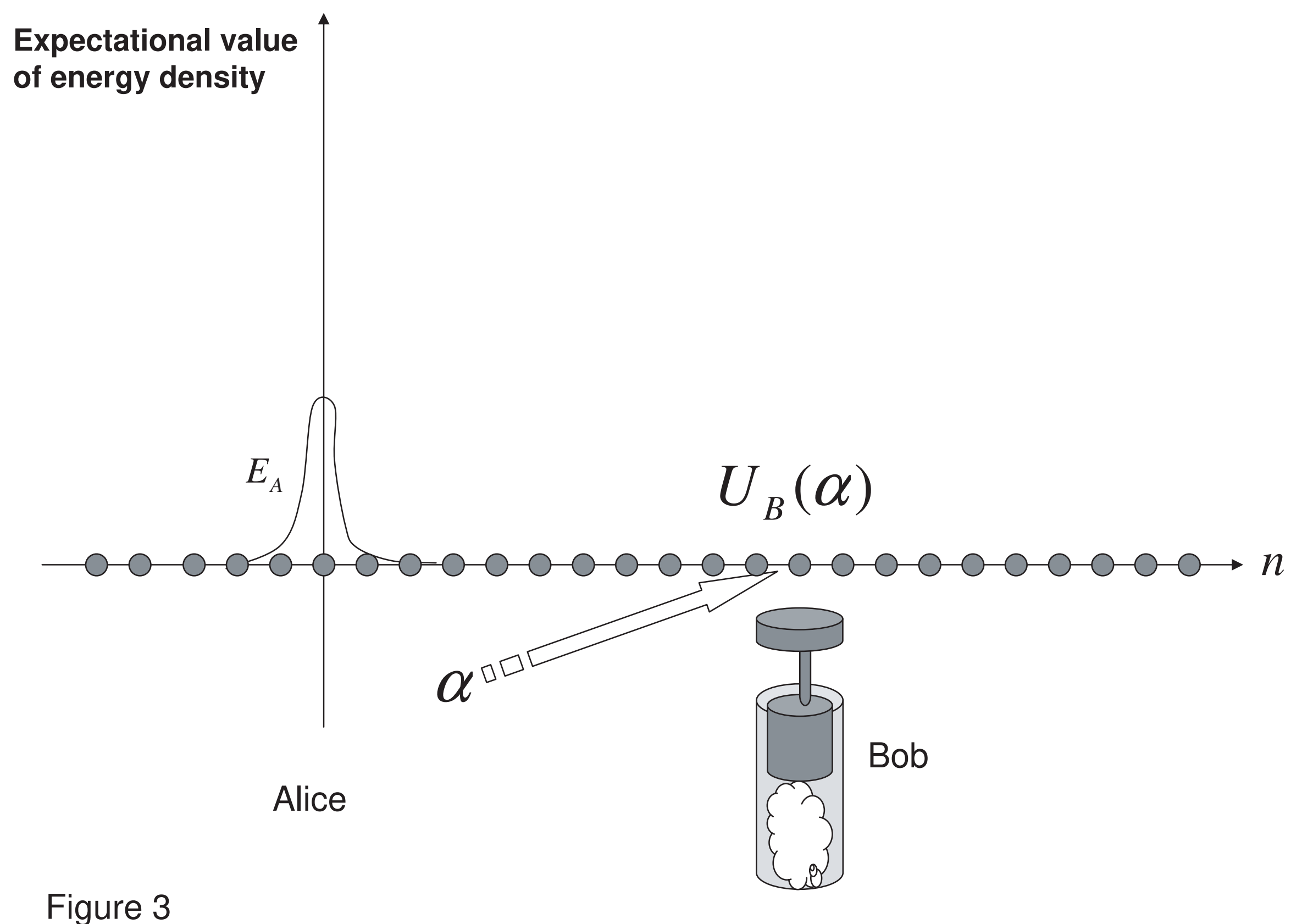




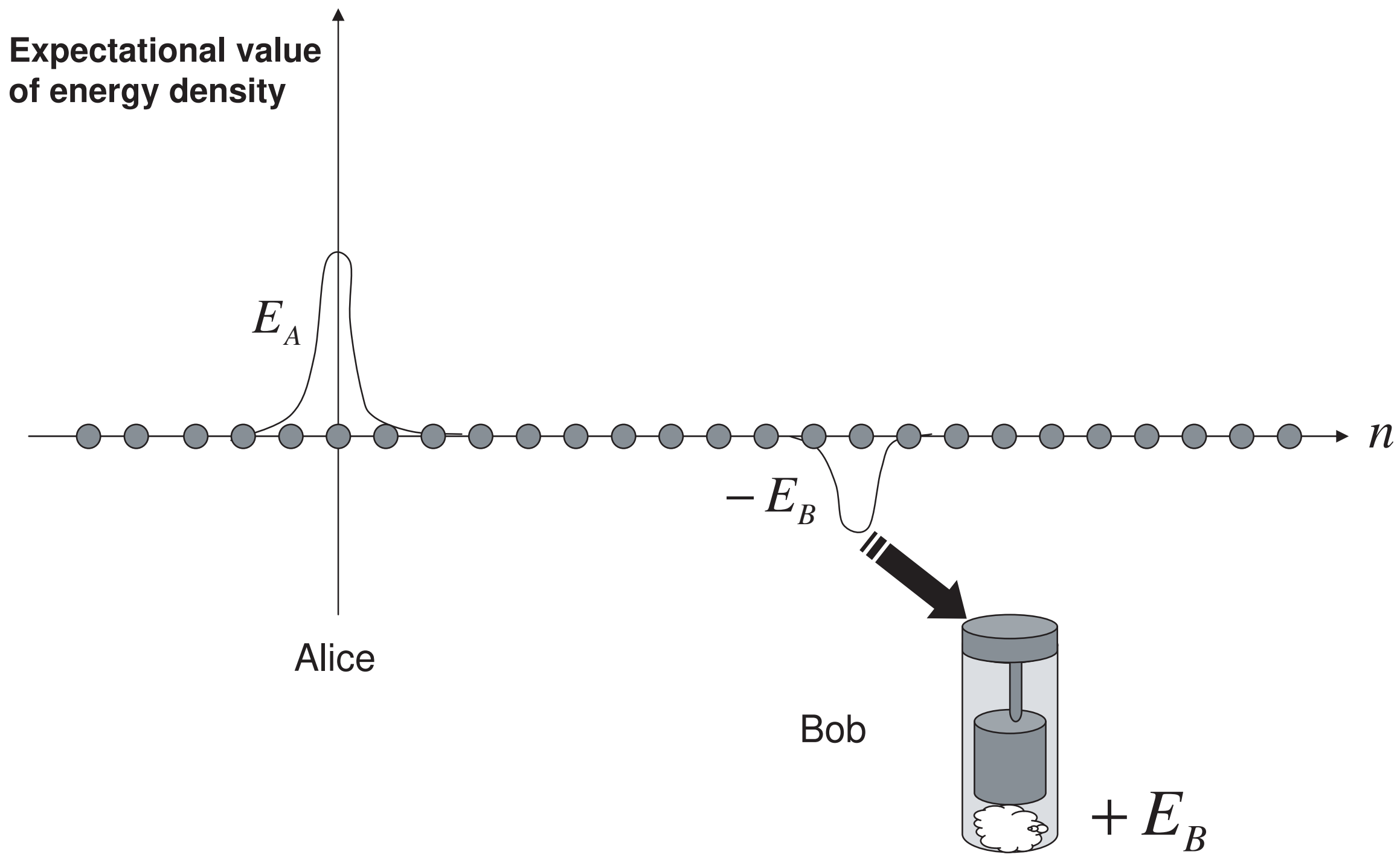

Figure 4 